\title{
Note on semiclassical states for the Schrödinger equation with nonautonomous nonlinearities
}

\author{
Bartosz Bieganowski \\ Nicolaus Copernicus University, Faculty of Mathematics and Computer Science, ul. Chopina 12/18, 87-100 Toruń, Poland \\ Jarosław Mederski \\ Institute of Mathematics, Polish Academy of Sciences, ul. Sniadeckich 8, 00-656 Warsaw, Poland, \\ CRC 1173 Wavephenomena: Analysis and Numerics, Departement of Mathematics, Karlsruhe Institute of Technology (KIT), \\ D-76128 Karlsruhe, Germany
}

\begin{abstract}
We consider the following Schrödinger equation

$$
-\hbar^{2} \Delta u+V(x) u=\Gamma(x) f(u) \text { in } \mathbb{R}^{N}
$$

where $u \in H^{1}\left(\mathbb{R}^{N}\right), u>0, \hbar>0$ and $f$ is superlinear and subcritical nonlinear term. We show that if $V$ attains local minimum and $\Gamma$ attains global maximum at the same point or $V$ attains global minimum and $\Gamma$ attains local maximum at the same point, then there exists a positive solution for sufficiently small $\hbar>0$.

Keywords: semiclassical limit, variational methods, bounded potential, nonautonomous nonlinearity 2010 MSC: 35Q55, 35A15, 35J20
\end{abstract}

\section{Introduction}

We consider the following semilinear elliptic problem

$$
-\hbar^{2} \Delta u+V(x) u=\Gamma(x) f(u) \text { in } \mathbb{R}^{N}, N \geq 1,
$$

where $u \in H^{1}\left(\mathbb{R}^{N}\right)$ and $u>0$. Equation (1.1) describes the so-called standing waves of the nonlinear, time-dependent Schrödinger equation of the form

$$
\mathrm{i} \hbar \frac{\partial \Psi}{\partial t}=-\frac{\hbar^{2}}{2 m} \Delta \Psi+V(x) \Psi-h(x, \Psi) .
$$

Solutions of (1.1) for sufficiently small $\hbar>0$ are called semiclassical states. Recently many papers have been devoted to study semiclassical states, see eg. [1 [5, 9, 10] and references therein and most of the concentrate on the case $\Gamma=1$. Our aim is to show that the method introduced by del Pino and Felmer in [4] can be also applied to a general class of problems with nonautonomous nonlinearities. Moreover, contrary to [4], we do not need the Hölder continuity of the potential $V$, since we do not use the regularity of solutions to show e.g. Lemma 2.3 below.

We impose the following condition on the potential $V$.

(V) $V \in L^{\infty}\left(\mathbb{R}^{N}\right)$ is continuous and there is a constant $\alpha>0$ such that $V(x) \geq \alpha$ for all $x \in \mathbb{R}^{N}$.

We assume that $f: \mathbb{R}_{+} \rightarrow \mathbb{R}$ is of $\mathcal{C}^{1}$-class and satisfies the following conditions.

(F1) $f(u)=o(u)$ as $u \rightarrow 0^{+}$.

(F2) $\lim _{u \rightarrow \infty} \frac{f(u)}{u^{p-1}}=0$ for some $2<p<2^{*}$, where $2^{*}=\frac{2 N}{N-2}$ for $N \geq 3$ and $2^{*}=+\infty$ otherwise.

(F3) There is $2<\theta \leq p$ such that $0<\theta F(u) \leq f(u) u$ for $u>0$, where $F(u):=\int_{0}^{u} f(s) d s$.

(F4) The function $u \mapsto \frac{f(u)}{u}$ is nondecreasing.

Email addresses: bartoszb@mat.umk.pl (Bartosz Bieganowski), jmederski@impan.pl (Jarosław Mederski) 
Now $\Gamma$ satisfies the following condition.

( $\Gamma) \Gamma \in L^{\infty}\left(\mathbb{R}^{N}\right)$ is continuous and there is a constant $\beta>0$ such that $\Gamma(x) \geq \beta>0$ for all $x \in \mathbb{R}^{N}$.

We introduce the following relation between $V$ and $\Gamma$.

( $\Lambda$ ) Assume that there is a bounded, nonempty domain (i.e. open and connected set) $\Lambda \subset \mathbb{R}^{N}$ such that

$\left(\Lambda_{1}\right) \Gamma$ is $\mathbb{Z}^{N}$-periodic and there is $x_{\min } \in \Lambda$ such that $V\left(x_{\min }\right)=\inf _{\Lambda} V<\min _{\partial \Lambda} V$ and $\Gamma\left(x_{\min }\right)=\sup _{\mathbb{R}^{N}} \Gamma$

or

$\left(\Lambda_{2}\right) V$ is $\mathbb{Z}^{N}$-periodic and there is $x_{\max } \in \Lambda$ such that $\Gamma\left(x_{\max }\right)=\sup _{\Lambda} \Gamma>\max _{\partial \Lambda} \Gamma \operatorname{and} V\left(x_{\max }\right)=\inf _{\mathbb{R}^{N}} V$.

Remark 1.1. Without loss of generality we may assume that $|\Gamma|_{\infty}=1$, where $|\cdot|_{k}$ denotes the usual $L^{k}$-norm with $k \geq 1$ or $k=\infty$. Indeed, see that

$$
\Gamma(x) f(u)=\frac{\Gamma(x)}{|\Gamma|_{\infty}}|\Gamma|_{\infty} f(u)
$$

Taking $\hat{\Gamma}(x):=\frac{\Gamma(x)}{|\Gamma|_{\infty}}$ and $\hat{f}(u):=|\Gamma|_{\infty} f(u)$ we see that all conditions are still satisfied and $|\hat{\Gamma}|_{\infty}=1$. Hence in the rest of the paper we take $|\Gamma|_{\infty}=1$.

Our main result reads as follows.

Theorem 1.2. Suppose that $(V),(F 1)-(F 4),(\Gamma)$ and $(\Lambda)$ are satisfied. Then there is $\hbar_{0}>0$ such that for any $\hbar \in\left(0, \hbar_{0}\right)$ the problem (1.1) has a positive solution $u \in H^{1}\left(\mathbb{R}^{N}\right) \cap \mathcal{C}\left(\mathbb{R}^{N}\right)$ and there are constants $C, \alpha>0$ such that $u(x) \leq C \exp (-\alpha|x|)$.

Define the energy functional $\mathcal{J}_{\hbar}: H \rightarrow \mathbb{R}$

$$
\mathcal{J}_{\hbar}(u):=\frac{1}{2} \int_{\mathbb{R}^{N}} \hbar^{2}|\nabla u|^{2}+V(x) u^{2} d x-\int_{\mathbb{R}^{N}} \Gamma(x) F(u) d x,
$$

where $H=H^{1}\left(\mathbb{R}^{N}\right)$. Note that for any fixed $\hbar>0$ the quadratic form

$$
H \ni u \mapsto Q_{\hbar}(u):=\int_{\mathbb{R}^{N}} \hbar^{2}|\nabla u|^{2}+V(x) u^{2} d x \in \mathbb{R}
$$

is positive definite. Hence for any $\hbar>0, u \mapsto \sqrt{Q_{\hbar}(u)}$ is well-defined norm on $H$ and equivalent to the classic one in $H$. Let $\theta$ be given by (F3) and fix $k>\frac{\theta}{\theta-2}>1$. In view of (F3) and (F4) there is $a>0$ such that $\frac{f(a)}{a}=\frac{\alpha}{k}$. We define

$$
\tilde{f}(u):= \begin{cases}f(u) & \text { for } u \leq a \\ \frac{\alpha}{k} u & \text { for } u>a\end{cases}
$$

where $\alpha$ has beed introduced in (V) and

$$
g(x, u):=\chi_{\Lambda}(x) \Gamma(x) f(u)+\left(1-\chi_{\Lambda}(x)\right) \Gamma(x) \tilde{f}(u) .
$$

In what follows we will consider $f$ as a function $f: \mathbb{R} \rightarrow \mathbb{R}$ satisfying (F1)-(F4) for on $\mathbb{R}_{+}$and defined as 0 for $u \leq 0$. Then $g: \mathbb{R}^{N} \times \mathbb{R} \rightarrow \mathbb{R}$ is a Carathéodory function. Moreover the following conditions hold.

(G1) $g(x, u)=o(u)$ for $|u| \rightarrow 0^{+}$uniformly in $x \in \mathbb{R}^{N}$.

(G2) $\lim _{u \rightarrow \infty} \frac{g(x, u)}{u^{p-1}}=0$ for some $2<p<2^{*}$ uniformly in $x \in \mathbb{R}^{N}$.

(G3) There is $2<\theta \leq p$ such that

$$
0<\theta G(x, u) \leq g(x, u) u \text { for } x \in \Lambda, u>0
$$

and

$$
0 \leq 2 G(x, u) \leq g(x, u) u \leq \frac{1}{k} V(x) u^{2} \quad \text { for } x \notin \Lambda, u>0
$$


(G4) The function $u \mapsto \frac{g(x, u)}{u}$ is nondecreasing on $(0, \infty)$ for all $x \in \mathbb{R}^{N}$. Moreover, if $x \in \mathbb{R}^{N} \backslash \Lambda$, the function $u \mapsto \frac{g(x, u)}{u}$ is constant on $[a, \infty)$.

Indeed, (G1), (G2) and (G4) are obvious and we need to check (G3). For $x \in \Lambda$ we have $g(x, u)=\Gamma(x) f(u)$ and $G(x, u)=\Gamma(x) F(u)$, so the statement follows from (F3). Fix $x \notin \Lambda$. From (G4) we have

$$
G(x, u)=\int_{0}^{u} \frac{g(x, s)}{s} s d s \leq \frac{g(x, u)}{u} \int_{0}^{u} s d s=\frac{1}{2} g(x, u) u .
$$

Hence $0 \leq 2 G(x, u) \leq g(x, u) u$. Moreover

$$
g(x, u) u=\Gamma(x) \tilde{f}(u) u= \begin{cases}\Gamma(x) \frac{f(u)}{u} u^{2} \leq \Gamma(x) \frac{\alpha}{k} u^{2}, & \text { for } u \leq a, \\ \Gamma(x) \frac{\alpha}{k} u^{2}, & \text { for } u>a .\end{cases}
$$

Thus

$$
g(x, u) u \leq \Gamma(x) \frac{\alpha}{k} u^{2} \leq \frac{1}{k} \Gamma(x) V(x) u^{2} \leq \frac{1}{k} V(x) u^{2}
$$

and the proof of (G3) is completed. Let $\Phi_{\hbar}: H \rightarrow \mathbb{R}$ be given by

$$
\Phi_{\hbar}(u):=\frac{\hbar^{2}}{2} \int_{\mathbb{R}^{N}}|\nabla u|^{2} d x+\frac{1}{2} \int_{\mathbb{R}^{N}} V(x) u^{2} d x-\int_{\mathbb{R}^{N}} G(x, u) d x .
$$

From [4, Lemma 2.1] we obtain the following.

Lemma 1.3. The functional $\Phi_{\hbar}$ possesses a positive critical point $u_{\hbar} \in H$ such that $\Phi_{\hbar}\left(u_{\hbar}\right)=c_{\hbar}$, where

$$
c_{\hbar}:=\inf _{u \in H \backslash\{0\}} \sup _{t \geq 0} \Phi_{\hbar}(t u) .
$$

Applying [8, Theorem 4.2] we see that $u_{\hbar}$ is continuous and exponentially decays at infinity. Define

$$
m_{\hbar}:=\max _{\partial \Lambda} u_{\hbar}
$$

\section{Case $\left(\Lambda_{1}\right)$}

Put $V_{0}:=\min _{\Lambda} V$. Let $w \in H$ be a least energy solution to $-\Delta w+V_{0} w=\Gamma(x) f(w)$, in particular

$$
\underline{c}:=I_{0}(w)=\inf _{v \in H \backslash\{0\}} \sup _{t \geq 0} I_{0}(t v)
$$

where

$$
I_{0}(v)=\frac{1}{2} \int_{\mathbb{R}^{N}}|\nabla v|^{2}+V_{0} v^{2} d x-\int_{\mathbb{R}^{N}} \Gamma(x) F(v) d x .
$$

Under $\left(\Lambda_{1}\right)$ we have that $\Gamma$ is $\mathbb{Z}^{N}$-periodic, hence the solution exists (see e.g. [8, 9]).

Lemma 2.1. There holds $\Phi_{\hbar}\left(u_{\hbar}\right) \leq \hbar^{N}(\underline{c}+o(1))$ as $\hbar \rightarrow 0^{+}$.

Proof. Let $x_{0} \in \Lambda$ be such that $V\left(x_{0}\right)=V_{0}$. Let $u(x):=w\left(\frac{x-x_{0}}{\hbar}\right)$. Then $\Phi_{\hbar}\left(u_{\hbar}\right) \leq \sup _{t>0} \Phi_{\hbar}(t u)=\Phi_{\hbar}\left(t_{0} u\right)$ for some $t_{0}>0$. See that

$$
\begin{aligned}
\Phi_{\hbar}\left(t_{0} u\right) & =\frac{t_{0}^{2} \hbar^{2}}{2} \int_{\mathbb{R}^{N}}|\nabla u|^{2} d x+\frac{t_{0}^{2}}{2} \int_{\mathbb{R}^{N}} V(x) u^{2} d x-\int_{\mathbb{R}^{N}} G\left(x, t_{0} u\right) d x \\
& =\hbar^{N}\left(I_{0}\left(t_{0} w\right)+\frac{t_{0}^{2}}{2} \int_{\mathbb{R}^{N}}\left[V\left(x_{0}+\hbar x\right)-V_{0}\right] w^{2} d x+\int_{\mathbb{R}^{N}} \Gamma(x) F\left(t_{0} w\right)-G\left(x_{0}+\hbar x, t_{0} w\right) d x\right) .
\end{aligned}
$$

From the Lebesgue's dominated convergence theorem we have $\int_{\mathbb{R}^{N}}\left[V\left(x_{0}+\hbar x\right)-V_{0}\right] w^{2} d x \rightarrow 0$. Note that $G\left(x, t_{0} w\right) \leq F\left(t_{0} w\right)$. Again, from the Lebesgue's dominated convergence theorem the following convergence hold

$$
\int_{\mathbb{R}^{N}} G\left(x_{0}+\hbar x, t_{0} w\right) d x \rightarrow \int_{\mathbb{R}^{N}} \Gamma\left(x_{0}\right) F\left(t_{0} w\right) d x
$$

Hence $\int_{\mathbb{R}^{N}} \Gamma(x) F\left(t_{0} w\right)-G\left(x_{0}+\hbar x, t_{0} w\right) d x \rightarrow \int_{\mathbb{R}^{N}} \Gamma(x) F\left(t_{0} w\right)-\Gamma\left(x_{0}\right) F\left(t_{0} w\right) d x \leq 0$ and finally

$$
\Phi_{\hbar}\left(t_{0} u\right) \leq \hbar^{N}\left(I_{0}\left(t_{0} w\right)+o(1)\right) \leq \hbar^{N}(\underline{c}+o(1))
$$


Lemma 2.2. There is $C>0$ such that

$$
\int_{\mathbb{R}^{N}} \hbar^{2}\left|\nabla u_{\hbar}\right|^{2}+V(x)\left|u_{\hbar}\right|^{2} d x \leq C \hbar^{N}
$$

Proof. Indeed, we have $\Phi_{\hbar}^{\prime}\left(u_{\hbar}\right)\left(u_{\hbar}\right)=0$, i.e. $\int_{\mathbb{R}^{N}} \hbar^{2}\left|\nabla u_{\hbar}\right|^{2}+V(x)\left|u_{\hbar}\right|^{2} d x=\int_{\mathbb{R}^{N}} g\left(x, u_{\hbar}\right) u_{\hbar} d x$. On the other hand

$$
\begin{aligned}
\frac{1}{2} \int_{\mathbb{R}^{N}} \hbar^{2}\left|\nabla u_{\hbar}\right|^{2}+V(x)\left|u_{\hbar}\right|^{2} d x & =\Phi_{\hbar}\left(u_{\hbar}\right)+\int_{\mathbb{R}^{N}} G\left(x, u_{\hbar}\right) d x \\
& \leq \hbar^{N}(\underline{c}+o(1))+\frac{1}{\theta} \int_{\Lambda} g\left(x, u_{\hbar}\right) u_{\hbar} d x+\frac{1}{2 k} \int_{\mathbb{R}^{N}} V(x)\left|u_{\hbar}\right|^{2} d x \\
& \leq C_{1} \hbar^{N}+\left(\frac{1}{\theta}+\frac{1}{2 k}\right) \int_{\mathbb{R}^{N}} \hbar^{2}\left|\nabla u_{\hbar}\right|^{2}+V(x)\left|u_{\hbar}\right|^{2} d x
\end{aligned}
$$

Hence

$$
\left(\frac{1}{2}-\frac{1}{\theta}-\frac{1}{2 k}\right) \int_{\mathbb{R}^{N}} \hbar^{2}\left|\nabla u_{\hbar}\right|^{2}+V(x)\left|u_{\hbar}\right|^{2} d x \leq C_{1} \hbar^{N} .
$$

Moreover $\frac{1}{2}-\frac{1}{\theta}-\frac{1}{2 k}=\frac{1}{2}\left(\frac{\theta-2}{\theta}-\frac{1}{k}\right)>0$ and the proof is finished.

Lemma 2.3. If $\hbar_{n} \rightarrow 0^{+}$and $\left(x_{n}\right) \subset \bar{\Lambda}$ are such that $u_{\hbar_{n}}\left(x_{n}\right) \geq b>0$, then $\lim _{n \rightarrow \infty} V\left(x_{n}\right)=V_{0}$.

Proof. Assume by contradiction, passing to a subsequence, that $x_{n} \rightarrow \bar{x} \in \bar{\Lambda}$ and $V(\bar{x})>V_{0}$. Put $v_{n}(x):=$ $u_{\hbar_{n}}\left(x_{n}+\hbar_{n} x\right)$. Obviously, $v_{n} \in H$ satisfies the equation

$$
-\Delta v_{n}+V\left(x_{n}+\hbar_{n} x\right) v_{n}=g\left(x_{n}+\hbar_{n} x, v_{n}\right) \quad \text { in } \mathbb{R}^{N} .
$$

From Lemma 2.2 it follows easily that $\left(v_{n}\right)$ is bounded in $H$ and therefore $v_{n} \rightarrow v$ in $H$ for some $v \in H$. Take any $\varphi \in \mathcal{C}_{0}^{\infty}\left(\mathbb{R}^{N}\right)$ and see that

$$
\int_{\mathbb{R}^{N}} \nabla v_{n} \cdot \nabla \varphi d x \rightarrow \int_{\mathbb{R}^{N}} \nabla v \cdot \nabla \varphi d x
$$

Moreover

$$
\int_{\mathbb{R}^{N}} V\left(x_{n}+\hbar_{n} x\right) v_{n} \varphi d x \rightarrow \int_{\mathbb{R}^{N}} V(\bar{x}) v \varphi d x
$$

Functions $\chi_{n}(x):=\chi_{\Lambda}\left(x_{n}+\hbar_{n} x\right)$ are bounded in $L_{\text {loc }}^{t}\left(\mathbb{R}^{N}\right)$ for any $1<t<\infty$, and therefore $\chi_{n} \rightarrow \chi$ in $L_{\text {loc }}^{t}\left(\mathbb{R}^{N}\right)$, where $0 \leq \chi \leq 1$. Hence $v \in H$ is a weak solution to

$$
-\Delta v+V(\bar{x}) v=\bar{g}(x, v) \text { in } \mathbb{R}^{N},
$$

where

$$
\bar{g}(x, u)=\chi(x) \Gamma(\bar{x}) f(u)+(1-\chi(x)) \Gamma(\bar{x}) \tilde{f}(u) .
$$

The associated energy functional is given by

$$
\overline{\mathcal{J}}(u):=\frac{1}{2} \int_{\mathbb{R}^{N}}|\nabla u|^{2}+V(\bar{x})|u|^{2} d x-\int_{\mathbb{R}^{N}} \bar{G}(x, u) d x \quad \text { for } u \in H^{1}\left(\mathbb{R}^{N}\right)
$$

and $\bar{G}(x, u):=\int_{0}^{u} \bar{g}(x, s) d s$. Since $v$ is a weak solution, we have $\overline{\mathcal{J}}^{\prime}(v)=0$. Set

$$
\mathcal{J}_{n}(u):=\frac{1}{2} \int_{\mathbb{R}^{N}}|\nabla u|^{2}+V\left(x_{n}+\hbar_{n} x\right)|u|^{2} d x-\int_{\mathbb{R}^{N}} G\left(x_{n}+\hbar_{n} x, u\right) d x \quad \text { for } u \in H^{1}\left(\mathbb{R}^{N}\right)
$$

and obviously $\mathcal{J}_{n}^{\prime}\left(v_{n}\right)=0$. See that

$$
\mathcal{J}_{n}\left(v_{n}\right)=\mathcal{J}_{n}\left(v_{n}\right)-\frac{1}{2} \mathcal{J}_{n}^{\prime}\left(v_{n}\right)\left(v_{n}\right)=\frac{1}{2} \int_{\mathbb{R}^{N}} g\left(x_{n}+\hbar_{n} x, v_{n}\right) v_{n}-2 G\left(x_{n}+\hbar_{n} x, v_{n}\right) d x .
$$

Define

$$
h_{n}:=g\left(x_{n}+\hbar_{n} \cdot v_{n}\right) v_{n}-2 G\left(x_{n}+\hbar_{n} \cdot, v_{n}\right) .
$$


In view of (G3) we have $h_{n} \geq 0$. Fix $R>0$. In view of compact embedding we have $v_{n} \rightarrow v$ in $L^{t}(B(0, R))$ for $t \in\left[2,2^{*}\right)$. Moreover $\chi_{n} \rightarrow \chi$ in any $L^{t}(B(0, R))$. Then

$$
\int_{B(0, R)} h_{n} d x \rightarrow \int_{B(0, R)} \bar{g}(x, v) v-2 \bar{G}(x, v) d x
$$

Hence, for every $\delta>0$ there is $R>0$ large enough such that

$$
\begin{aligned}
\frac{1}{2} \int_{\mathbb{R}^{N}} h_{n} d x & \geq \frac{1}{2} \int_{B(0, R)} h_{n} d x \rightarrow \frac{1}{2} \int_{B(0, R)} \bar{g}(x, v) v-2 \bar{G}(x, v) d x \\
& \geq \frac{1}{2} \int_{\mathbb{R}^{N}} \bar{g}(x, v) v-2 \bar{G}(x, v) d x-\delta=\overline{\mathcal{J}}(v)-\frac{1}{2} \overline{\mathcal{J}}^{\prime}(v)(v)-\delta=\overline{\mathcal{J}}(v)-\delta .
\end{aligned}
$$

Therefore $\lim \inf _{n \rightarrow \infty} \mathcal{J}_{n}\left(v_{n}\right) \geq \overline{\mathcal{J}}(v)$. On the other hand $\mathcal{J}_{n}\left(v_{n}\right)=\hbar_{n}^{-N} \Phi_{\hbar_{n}}\left(u_{\hbar_{n}}\right) \leq \underline{c}+o(1)$. Hence $\overline{\mathcal{J}}(v) \leq \underline{c}$. Taking into account that $f(u) \geq \tilde{f}(u)$ we have

$$
\overline{\mathcal{J}}(v)=\max _{\tau \geq 0} \overline{\mathcal{J}}(\tau v) \geq \inf _{u \in H^{1}\left(\mathbb{R}^{N}\right), u \neq 0} \sup _{\tau>0} I(\tau u)=: \bar{c},
$$

where

$$
I(u):=\frac{1}{2} \int_{\mathbb{R}^{N}}|\nabla u|^{2}+V(\bar{z}) u^{2} d x-\int_{\mathbb{R}^{N}} \Gamma(\bar{x}) F(u) d x .
$$

On the other hand, taking into account that $V(\bar{x})>V_{0}$, there is $\bar{c}>\underline{c}-$ a contradiction.

Lemma 2.4. There holds $\lim _{\hbar \rightarrow 0^{+}} m_{\hbar}=0$, where $m_{\hbar}$ is given by (1.2).

Proof. Assume by contradiction that $m_{\hbar} \nrightarrow \rightarrow 0$. Let $x_{\hbar} \in \partial \Lambda \subset \bar{\Lambda}$ be such that $u_{\hbar}\left(x_{\hbar}\right)=m_{\hbar}$. Then, up to a subsequence we have $u_{\hbar_{n}}\left(x_{\hbar_{n}}\right) \geq b>0$ and $x_{\hbar_{n}} \rightarrow x_{0} \in \partial \Lambda$. Hence, in view of Lemma 2.3 we gets

$$
\min _{\partial \Lambda} V \leq V\left(x_{0}\right)=V_{0}=\inf _{\Lambda} V<\min _{\partial \Lambda} V,
$$

which is a contradiction.

\section{Case $\left(\Lambda_{2}\right)$}

Put $\Gamma_{0}:=\max _{\Lambda} \Gamma$. Let $w \in H$ be a least energy solution to $-\Delta w+V(x) w=\Gamma_{0} f(w)$, in particular

$$
\underline{c}:=I_{0}(w)=\inf _{v \in H \backslash\{0\}} \sup _{t \geq 0} I_{0}(t v),
$$

where

$$
I_{0}(v)=\frac{1}{2} \int_{\mathbb{R}^{N}}|\nabla v|^{2}+V(x) v^{2} d x-\int_{\mathbb{R}^{N}} \Gamma_{0} F(v) .
$$

Under $\left(\Lambda_{2}\right)$ we have that $V$ is $\mathbb{Z}^{N}$-periodic, hence the solution exists (see e.g. [8, 9$]$ ).

Lemma 3.1. There holds $\Phi_{\hbar}\left(u_{\hbar}\right) \leq \hbar^{N}(\underline{c}+o(1))$ as $\hbar \rightarrow 0^{+}$.

Proof. Let $x_{\max } \in \Lambda$ be such that $\Gamma\left(x_{\max }\right)=\Gamma_{0}$. Let $u(x):=w\left(\frac{x-x_{\max }}{\hbar}\right)$. Then $\Phi_{\hbar}\left(u_{\hbar}\right) \leq \sup _{t>0} \Phi_{\hbar}(t u)=$ $\Phi_{\hbar}\left(t_{0} u\right)$ for some $t_{0}>0$. See that

$$
\begin{aligned}
\Phi_{\hbar}\left(t_{0} u\right) & =\frac{t_{0}^{2} \hbar^{2}}{2} \int_{\mathbb{R}^{N}}|\nabla u|^{2} d x+\frac{t_{0}^{2}}{2} \int_{\mathbb{R}^{N}} V(x) u^{2} d x-\int_{\mathbb{R}^{N}} G\left(x, t_{0} u\right) d x \\
& =\hbar^{N}\left(I_{0}\left(t_{0} w\right)+\frac{t_{0}^{2}}{2} \int_{\mathbb{R}^{N}}\left[V\left(x_{\max }+\hbar x\right)-V(x)\right] w^{2} d x+\int_{\mathbb{R}^{N}} \Gamma_{0} F\left(t_{0} w\right)-G\left(x_{\max }+\hbar x, t_{0} w\right) d x\right) .
\end{aligned}
$$

From the Lebesgue's dominated convergence theorem we have

$$
\int_{\mathbb{R}^{N}}\left[V\left(x_{\max }+\hbar x\right)-V(x)\right] w^{2} d x \rightarrow \int_{\mathbb{R}^{N}}\left[V\left(x_{\max }\right)-V(x)\right] w^{2} d x \leq 0 .
$$

Note that $G\left(x, t_{0} w\right) \leq F\left(t_{0} w\right)$. Again, from the Lebesgue's dominated convergence theorem the following convergence hold

$$
\int_{\mathbb{R}^{N}} \Gamma_{0} F\left(t_{0} w\right)-G\left(x_{\max }+\hbar x, t_{0} w\right) d x \rightarrow \int_{\mathbb{R}^{N}} \Gamma_{0} F\left(t_{0} w\right)-\Gamma\left(x_{\max }\right) F\left(t_{0} w\right) d x=0 .
$$

Finally $\Phi_{\hbar}\left(t_{0} u\right) \leq \hbar^{N}\left(I_{0}\left(t_{0} w\right)+o(1)\right) \leq \hbar^{N}(\underline{c}+o(1))$.

Now we can repeat the proof of Lemma 2.2, 2.3 and 2.4. 


\section{Conclusion}

Proof of Theorem 1.2. Let $u_{\hbar}$ be a positive critical point for $\Phi_{\hbar}$. In view of Lemma 2.4 there is $\hbar_{0}$ such that for any $\hbar \in\left(0, \hbar_{0}\right)$ there holds $m_{\hbar}<a$. Therefore $u_{\hbar}(x)<a$ for $x \in \partial \Lambda$. Hence, in view of the maximum principle, we have

$$
u_{\hbar}(x) \leq a \quad \text { for } x \in \Lambda \text {. }
$$

Take $\left(u_{\hbar}-a\right)_{+}:=\max \left\{u_{\hbar}-a, 0\right\}$ as a test function for $\Phi_{\hbar}$, i.e. we have $\Phi_{\hbar}^{\prime}\left(u_{\hbar}\right)\left(\left(u_{\hbar}-a\right)_{+}\right)=0$. Thus we get

$$
\int_{\mathbb{R}^{N} \backslash \Lambda} \hbar^{2}\left|\nabla\left(u_{\hbar}-a\right)_{+}\right|^{2}+c(x)\left(u_{\hbar}-a\right)_{+}^{2}+c(x) a\left(u_{\hbar}-a\right)_{+} d x=0,
$$

where $c(x)=V(x)-\frac{g\left(x, u_{\hbar}(x)\right)}{u_{\hbar}(x)}$. Moreover, for $x \in \mathbb{R}^{N} \backslash \Lambda$, taking into account that $|\Gamma|_{\infty}=1$, we obtain $\frac{g\left(x, u_{\hbar}(x)\right)}{u_{\hbar}(x)} \leq \frac{\alpha}{k}$. Therefore $c(x)>0$ for $x \in \mathbb{R}^{N} \backslash \Lambda$. Hence all summands in (4.1) are zero. In particular $\left(u_{\hbar}-a\right)_{+}=0$ and $u_{\hbar}(x) \leq a$ for $x \in \mathbb{R}^{N} \backslash \Lambda$. Hence $g\left(x, u_{\hbar}(x)\right)=\Gamma(x) f\left(u_{\hbar}(x)\right)$ and $u_{\hbar}$ is a solution of (1.1).

Acknowledgements. Bartosz Bieganowski was partially supported by the National Science Centre, Poland (Grant No. 2017/25/N/ST1/00531). Jarosław Mederski was partially supported by the National Science Centre, Poland (Grant No. 2014/15/D/ST1/03638) and by the Deutsche Forschungsgemeinschaft (DFG) through CRC 1173.

\section{References}

\section{References}

[1] J. Byeon, L. Jeanjean: Standing Waves for Nonlinear Schrödinger Equations with a General Nonlinearity, Arch. Rational Mech. Anal. 185 (2007), 185-200.

[2] J. Byeon, K. Tanaka: Semi-classical standing waves for nonlinear Schrödinger equations at structurally stable critical points of the potential, J. Eur. Math. Soc. 15 (2013), 1859-1899.

[3] P. d'Avenia, A. Pomponio, D. Ruiz: Semiclassical states for the nonlinear Schrödinger equation on saddle points of the potential via variational methods, J. Funct. Anal. 262 (2012), 4600-4633.

[4] M. del Pino, P.L. Felmer: Local mountain passes for semilinear elliptic problems in unbounded domains, Calc. Var. 4 (1996), 121-137.

[5] M. del Pino, P.L. Felmer: Semi-classical States for Nonlinear Schroödinger Equations, Journal of Functional Analysis 149 (1997), 245-265.

[6] Y. Ding, C. Lee, F. Zhao: Semiclassical limits of ground state solutions to Schrödinger systems, Calc. Var. 51 (2014), 725-760.

[7] Y. Ding, J. Wei: Semiclassical states for nonlinear Schrödinger equations with sign-changing potentials, J. Funct. Anal. 251 (2007), 546-572.

[8] J. Mederski: Ground states of a system of nonlinear Schrödinger equations with periodic potentials, Comm. Partial Differential Equations 41 (2016), no. 9, 1426-1440.

[9] P. Rabinowitz: On a class of nonlinear Schrödinger equations, Z. Angew. Math. Phys. 43 (1992), 270291.

[10] X. Wang: On Concentration of Positive Bound States of Nonlinear Schrödinger Equations, Commun. Math. Phys. 153 (1993), 229-244. 